\title{
Modular signature of long non-coding RNA association networks as a prognostic biomarker in lung cancer
}

\author{
Albert Li ${ }^{1}$, Wen-Hsuan Yu', Chia-Lang Hsu ${ }^{2}$, Hsuan-Cheng Huang ${ }^{3 *}$ and Hsueh-Fen Juan ${ }^{1,4,5^{*}}$ (i)
}

From The 19th Asia Pacific Bioinformatics Conference (APBC 2021) Tainan, Taiwan. 3-5 February 2021

\begin{abstract}
Background: Increasing amount of long non-coding RNAs (IncRNAs) have been found involving in many biological processes and played salient roles in cancers. However, up until recently, functions of most IncRNAs in lung cancer have not been fully discovered, particularly in the co-regulated IncRNAs. Thus, this study aims to investigate roles of IncRNA modules and uncover a module-based biomarker in lung adenocarcinoma (LUAD).

Results: We used gene expression profiles from The Cancer Genome Atlas (TCGA) to construct the IncRNA association networks, from which the highly-associated IncRNAs are connected as modules. It was found that the expression of some modules is significantly associated with patient's survival, including module N1 ( $H R=0.62,95 \%$ $\mathrm{Cl}=0.46-0.84, p=0.00189) ; \mathrm{N} 2(\mathrm{HR}=0.68, \mathrm{Cl}=0.50-0.93, p=0.00159) ; \mathrm{N} 4(\mathrm{HR}=0.70, \mathrm{Cl}=0.52-0.95, p=0.0205)$ and P3 $(\mathrm{HR}=0.68, \mathrm{Cl}=0.50-0.92, p=0.0123)$. The IncRNA signature consisting of these four prognosis-related modules, a 4-modular InCRNA signature, is associated with favourable prognosis in TCGA-LUAD ( $H R=0.51, C l=0.37-0.69, p$ value $=2.00 \mathrm{e}-05$ ). Afterwards, to assess the performance of the generic modular signature as a prognostic biomarker, we computed the time-dependent area under the receiver operating characteristics (AUC) of this 4-modular IncRNA signature, which showed AUC equals $68.44 \%$ on 336th day. In terms of biological functions, these modules are correlated with several cancer hallmarks and pathways, including Myc targets, E2F targets, cell cycle, inflammation/ immunity-related pathways, androgen/oestrogen response, KRAS signalling, DNA repair and epithelial-mesenchymal transition (EMT).

Conclusion: Taken together, we identified four novel LUAD prognosis-related IncRNA modules, and assessed the performance of the 4-modular IncRNA signature being a prognostic biomarker. Functionally speaking, these modules involve in oncogenic hallmarks as well as pathways. The results unveiled the co-regulated IncRNAs in LUAD and may provide a framework for further IncRNA studies in lung cancer.
\end{abstract}

Keywords: LncRNA association networks, Lung cancer, IncRNA modular prognostic biomarkers

*Correspondence: hsuancheng@nycu.edu.tw; yukijuan@ntu.edu.tw 1 Graduate Institute of Biomedical Electronics and Bioinformatics, National Taiwan University, Taipei 10617, Taiwan

${ }^{3}$ Institute of Biomedical Informatics, National Yang Ming Chiao Tung University, Taipei 11221, Taiwan

Full list of author information is available at the end of the article

\section{Background}

Lung cancer is one of the leading causes of cancer mortality around the globe [1]. Although a few oncogenic alterations can be targeted by certain medications, which showed greater therapeutic efficacy than chemotherapy $[2,3]$, the prognostic biomarkers assessing lung cancer 
patients are rather limited currently [3]. Therefore, further exploration in biomarkers that assess the prognosis of lung cancer patients is warranted.

LncRNAs, transcripts with length greater than 200 nucleotides [4], regulate gene expression through various mechanisms, including cis- [5-7], trans-[8, 9], and posttranscriptional regulation. Aberrant lncRNA regulations were found in various cancers [10]. These perturbations in transcriptome can be revealed by lncRNA-mRNA coexpression networks, resulting in the discovery of several key IncRNAs and mRNAs that contributed to cancer progression [11, 12]. Subsequent in silico and clinical studies further suggested that multiple lncRNAs can be integrated into singular signatures to better predict cancer patients' survival [13-16], tumour relapse $[17,18]$ and response to immunotherapy [15]. Given that the underlying biological explanation of combining multiple lncRNAs into one signature may still be unclear, it was speculated that functionally similar lncRNA may coappear within cells. Therefore, identifying highly associated lncRNAs and their roles in LUAD is crucial.

A lncRNA-mRNA co-expression network is a bi-partite network. The association between each lncRNA can be measured in a IncRNA-mRNA bi-partite network via computing the association index, a quantitative metric measuring the similarity between two lncRNAs according to the overlapping correlated mRNAs. By constructing the lncRNA association networks, similar lncRNAs would be clustered as modules [19]. Functions of the lncRNA modules can then be deduced by the modulecorrelated mRNAs under the norm of 'guilt by association' [20].

However, our understanding regarding the associated IncRNAs in LUAD has been primitive. The roles of LUAD-specific modules are to be confirmed. Furthermore, whether lncRNA modules signature can be a decent prognostic biomarker needs further investigation. Hence, we postulated that lncRNA modules are composed of co-regulated lncRNAs, and these modules play crucial roles in LUAD. To validate the hypothesis, we gathered gene expression profile from TCGA-LUAD project, constructed the lncRNA association networks, deduced impact of modules on patients' survival, and analysed biological roles of these lncRNA modules. In this study, we provided a module-based approach to uncover a novel biomarker in LUAD.

\section{Results}

\section{Overview of the analytical pipeline}

We proposed a computational pipeline (Fig. 1) to construct LUAD-specific lncRNA association networks and identify prognosis-related lncRNA modules. Firstly, the correlation between lncRNA and mRNA was quantified with Spearman correlation coefficient (SCC), which was then transformed to mutual rank (MR) index. The mRNAs within top- and bottom- MR index were selected as positive- and negative- correlated mRNAs respectively. Second, the association between each lncRNA was quantified by the association index. Next, we selected lncRNA pairs with top-scoring association index and constructed lncRNA association networks. Finally, we assessed roles of lncRNA modules in LUAD through survival analysis, time-dependent AUC, and functional enrichment analysis.

\section{Construction of the IncRNA association networks}

The gene expression profile of 553 LUAD samples were obtained from TCGA. After pre-processing, 4342 lncRNAs and 16,618 mRNAs remained. We quantified the correlation between lncRNAs and mRNAs with SCC (Fig. 2A). To identify the lncRNA-correlated mRNAs, SCCs were transformed to MR index [21]. The mRNAs with top and bottom $0.1 \%$ of MR index $(M R \leq \sqrt{4342 \times 16619} \times 0.001 \cong 8.49)$ were selected as the lncRNA- positively or negatively correlated mRNAs. The number of co-expressed mRNA of each lncRNA was shown in Fig. 2B. The mRNAs that are highly correlated with lncRNAs were chosen to calculate the association index between lncRNAs (Fig. 2C). Several association indices are popular and commonly used, such as Jaccard index. We used Pearson Correlation Coefficient (PCC) in this study because PCC can group the associated genes $(\mathrm{AUC}=0.903)$ and can best separate highly co-expressed gene pairs from the others $(\mathrm{AUC}=0.750)[20]$. We collected the lncRNA-lncRNA pairs having PCCs $>0.7$ and constructed the lncRNA association networks (LAN). The LAN constructed from the positively or negatively correlated mRNAs were coined as positive lncRNA association network (PLAN) or negative lncRNA association network (NLAN), respectively (Fig. 3).

\section{Characteristics of the IncRNA association networks}

To verify the scale-free property of generic lncRNA association networks, we inspected the relation between the degree $(k)$ of nodes and the degree density ( $p k)$ (Fig. 4A). The power law distribution was revealed when plotting $k$ against $p k$. Further, when taking the logarithm on both axes, the regression line significantly fit the data points, with the slope coefficient $=-1.96, p$ value $=0.000292$ on PLAN; the slope coefficient $=-2.24, p$ value $=6.51 \mathrm{e}-05$ on NALN. (Fig. 4B). The above results suggested that the lncRNA association networks were non-random, and had the scale-free property. We selected modules with nodes no less than 12 for further analysis (Fig. 4C). Six modules were selected from the NLAN, and three modules were from PLAN (Additional file 1: Table S1). Characteristics 
Data Retrieval

IncRNA and mRNA
expression profile
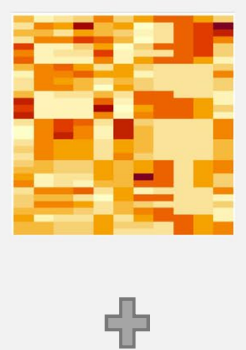

\section{Clinical survival information}

Network Construction

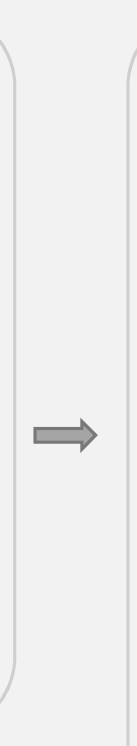

mRNA

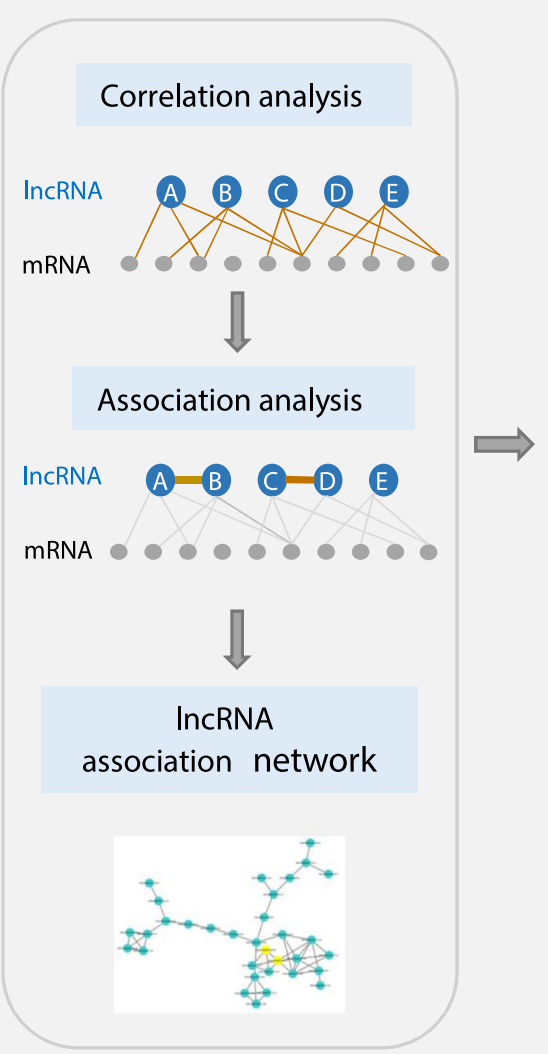

Functional Analysis

\section{Survival analysis}

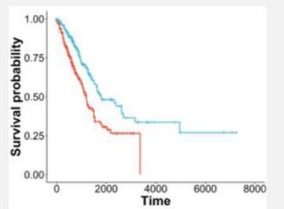

timeROC

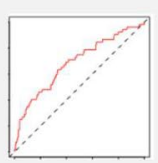

Functional enrichment

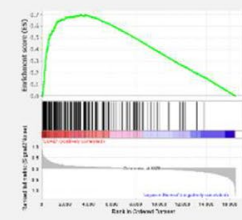

Fig. 1 The flowchart of prognostic IncRNAs module discovery in LUAD. The expression profile of IncRNA and mRNA as well as clinical data were retrieved from TCGA. To determine the IncRNA-correlated mRNAs, the IncRNA-mRNA correlation was quantified by SCC and MR. The IncRNA association was computed by PCC. The IncRNA pairs with PCC $>0.7$ were selected to construct the IncRNA association networks. LncRNAs that affect prognosis were selected and pooled to evaluate the impact IncRNA modules on survival. To evaluate the performance of generic modular IncRNA signature, we conducted time-AUC analysis. Finally, functional enrichment analysis was used to reveal potential mechanisms that IncRNA modules involved in. LUAD, lung adenocarcinoma; TCGA, the cancer genome atlas; SCC, Spearman's rank correlation coefficient; MR, mutual rank; PCC, Pearson correlation coefficient

of each module were visualized with box plots, including the expression of IncRNAs (Additional file 1: Fig. S1A), node degree (Additional file 1: Fig. S1B), clustering coefficient (Additional file 1: Fig. S1C), and betweenness centrality (Additional file 1: Fig. S1D).

\section{LncRNAs within the modules are highly associated}

Since we postulated that modules are composed of highly associated lncRNAs, we compared the association index, PCC, between intra- and inter-module lncRNAs. It was found that intra-module PCCs are significantly higher than that in inter-module PCCs (Fig. 5A, B), implying
lncRNAs within the modules are more alike. The PCC of each lncRNA pair was then visualized with a heat map (Fig. 5C, D). It can be seen that lncRNAs within the same modules are highly associated. The above findings suggested that the association index is can group highly associated lncRNAs together as modules.

\section{LncRNA modules are associated with the prognosis of LUAD patients}

To confirm the effects of the modules on patients' prognosis, we firstly screened the lncRNAs in the selected nine modules by univariate survival analysis (log-rank 

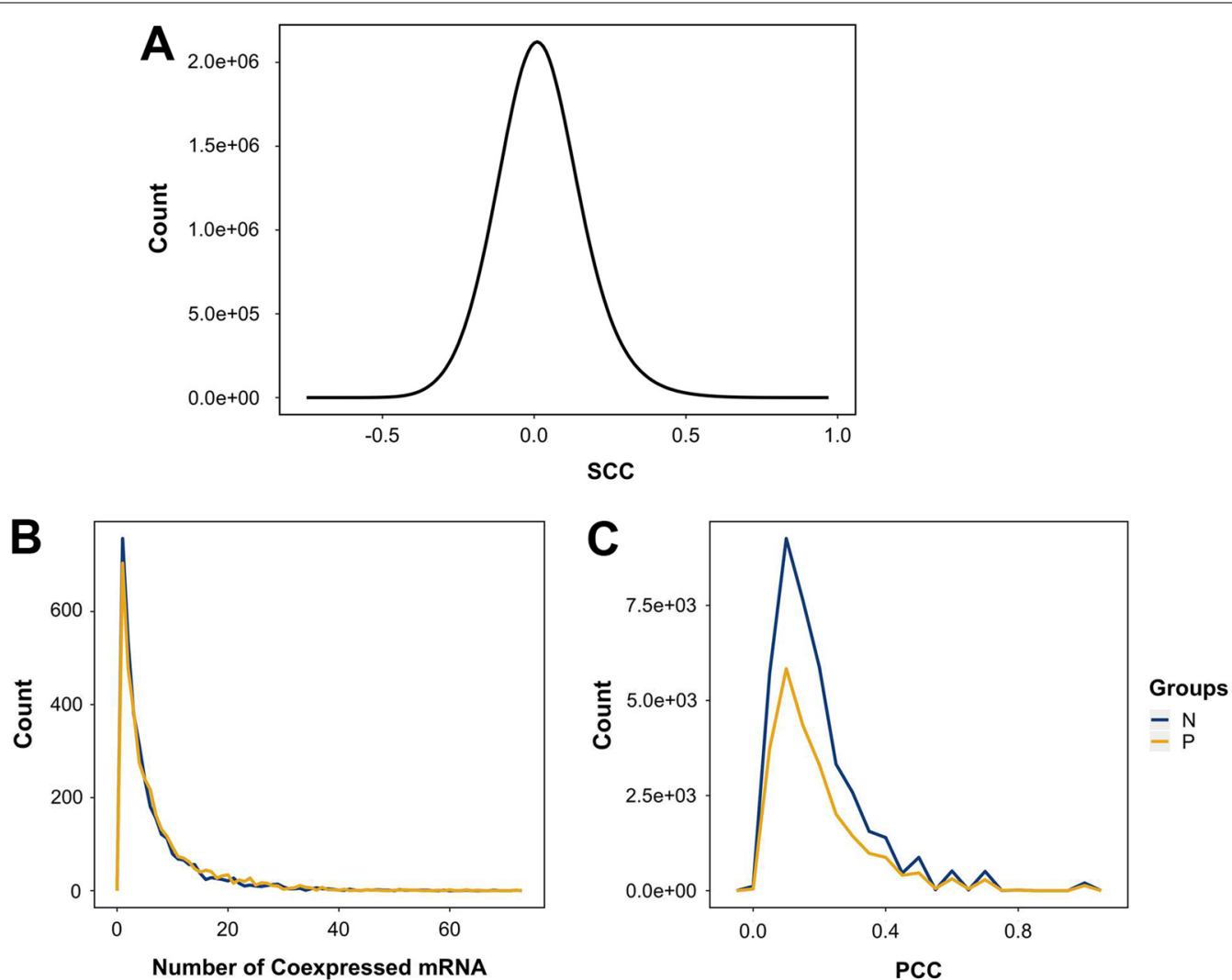

Fig. 2 Identification of correlated IncRNA-mRNA pairs and associated IncRNAs. A The distribution of the SCCs of IncRNA-mRNA pairs. B The distribution of the number of correlated mRNAs per IncRNA. The IncRNA-positively correlated mRNAs are defined as top $0.1 \%$ of mutual rank index (yellow) of IncRNA-mRNA pairs; the IncRNA-negatively correlated mRNAs are defined as bottom $0.1 \%$ of mutual rank index (blue) of IncRNA-mRNA pairs. C The PCC between each IncRNA was calculated considering the number of their correlated mRNAs. The distribution of PCC according to the IncRNA-positively correlated mRNAs (blue) and IncRNA-negatively correlated mRNAs (yellow) were plotted. N, measurement according to IncRNA-negatively correlated mRNAs; P, measurement according to IncRNA-positively correlated mRNAs; SCC, spearman's rank correlation coefficient

test). Because the results from univariate analysis may be confounded by factors that affect the outcome, we next utilised multivariate Cox proportional hazard model to adjust the cancer stage and the age of patients. Afterwards, we excluded the survival-related IncRNAs that were cancer-stage independent or not differentially expressed between cancer and normal tissue (see Additional file 1: Fig. S2). Finally, nine prognosis-related lncRNAs were selected (Table 1). Specifically, there are five prognostic lncRNAs (AC073655.2, AL031775.2, AL122010.1, AC079210.1, and AL391834.2) in module
N1, two lncRNAs (AL683813.2 and AC005759.1) in N2, one lncRNA (AC008937.3) in N4, and one lncRNA (AC099343.4) in P3. The modular expression was calculated by summing the coefficient-adjusted expression of the prognostic lncRNA(s). These four lncRNA modules are significantly associated with overall survival (OS) as shown in the Kaplan-Meier plots (Fig. 6A-D). We also inspected the impact of IncRNA modules on patients' progression free survival (PFS) (Fig. 6E-G). When adjusting the effects of cancer stage and patients' age on survival, the association between OS and IncRNA module is

(See figure on next page.)

Fig. 3 The IncRNA association networks. A The IncRNA-IncRNA pairs with PCC greater than 0.7 were selected to construct the positive IncRNA association network (PLAN). The calculation of PCC is based on the mRNAs that are positively correlated with IncRNAs. Modules P1-P3 (green square) are selected for following analyses. B As the above method, we measured the PCC based on the mRNAs that are negatively correlated with IncRNAs. The IncRNA pairs with PCC greater than 0.7 were collected to construct the negative IncRNA association network (NLAN). N1-N6 are modules selected for following analyses. C The number of nodes in each selected module 


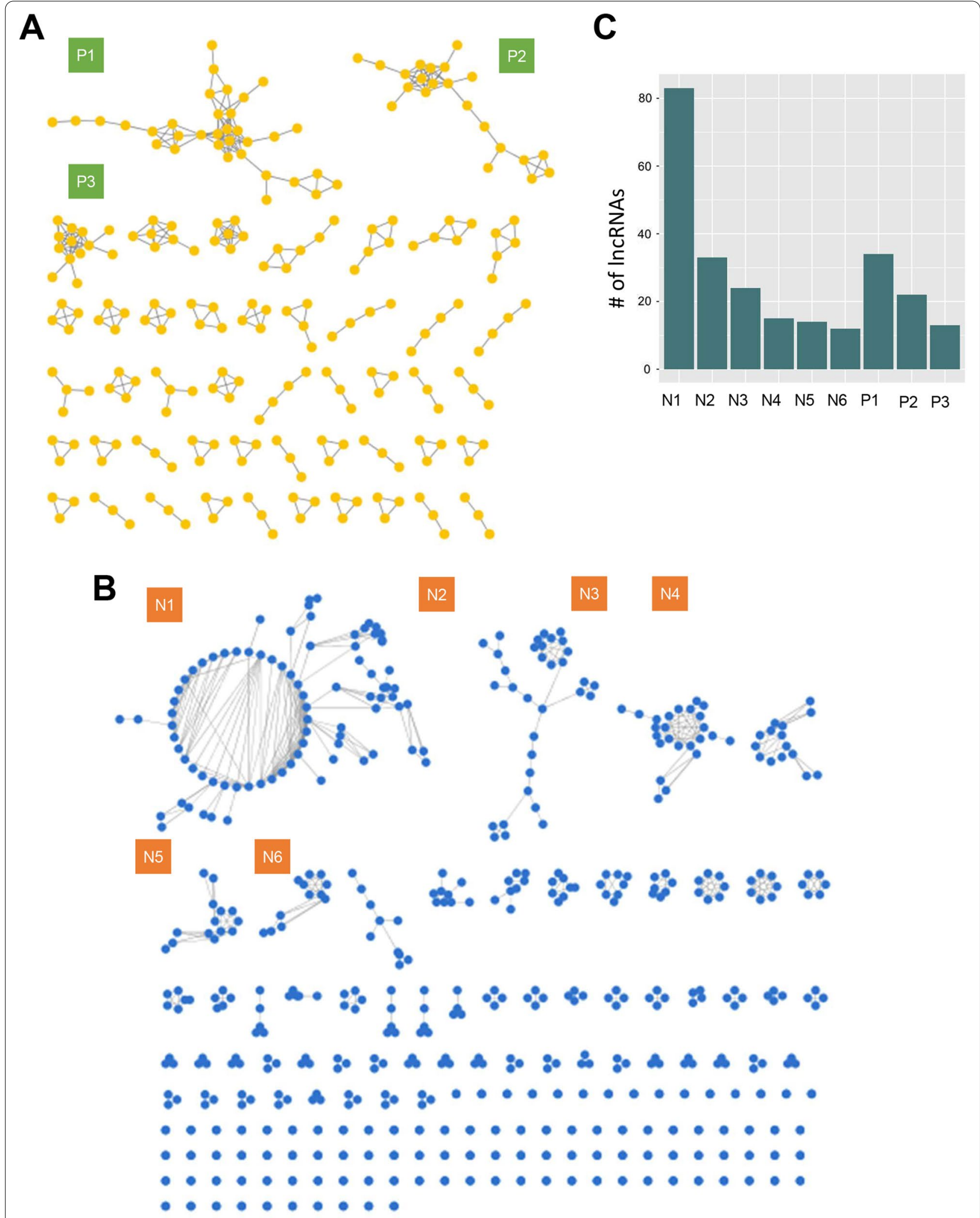

Fig. 3 (See legend on previous page.) 

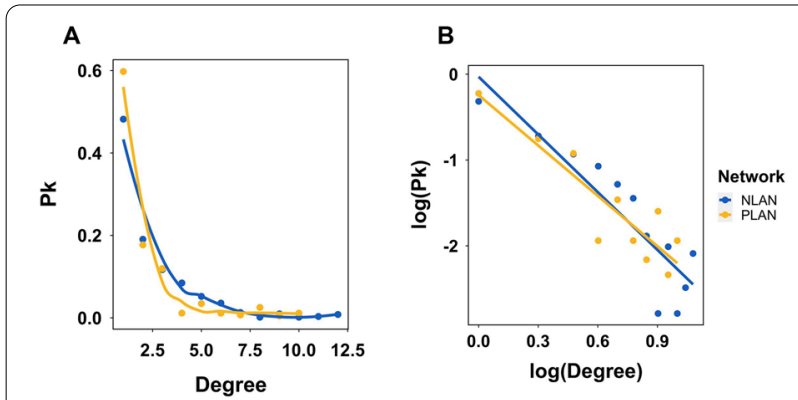

Fig. 4 Identification of the scale-free property of the IncRNA association networks. A The degree of each node in the networks were plotted against the degree density (pk). The fitted curve showed power law distribution. $\mathbf{B}$ The value in both axes underwent logarithm transformation. The fitted regression lines were significant, with $p=6.51 \mathrm{e}-05$ in NALN and $p=0.000292$ in PLAN respectively still significant. (Fig. 7A). Next, since combining multiple lncRNAs into one signature can better predict the outcomes $[13,14,16]$, four lncRNA modules $(\mathrm{N} 1, \mathrm{~N} 2$, N4, and P3) were pooled into one lncRNA signature. The OS $(p<0.0001)$ and PFS $(p=0.021)$ are significant (Fig. 7B, $C)$. The hazard ratio of the 4-module signature in the multivariable model is $0.51(95 \% \mathrm{CI}=0.37,0.69)$, and $p$ value $=2.00 \mathrm{e}-05$ (Fig. 7A).

\section{Assessment of the IncRNA modular signature as a novel prognostic biomarker in LUAD}

To assess the lncRNA modules as a prognostic biomarker in LUAD, we interrogated patients' OS and compared the AUC at several time intervals (Fig. 7D). The results were consistent with the former survival analyses. The 4-module lncRNA signature is better than individual
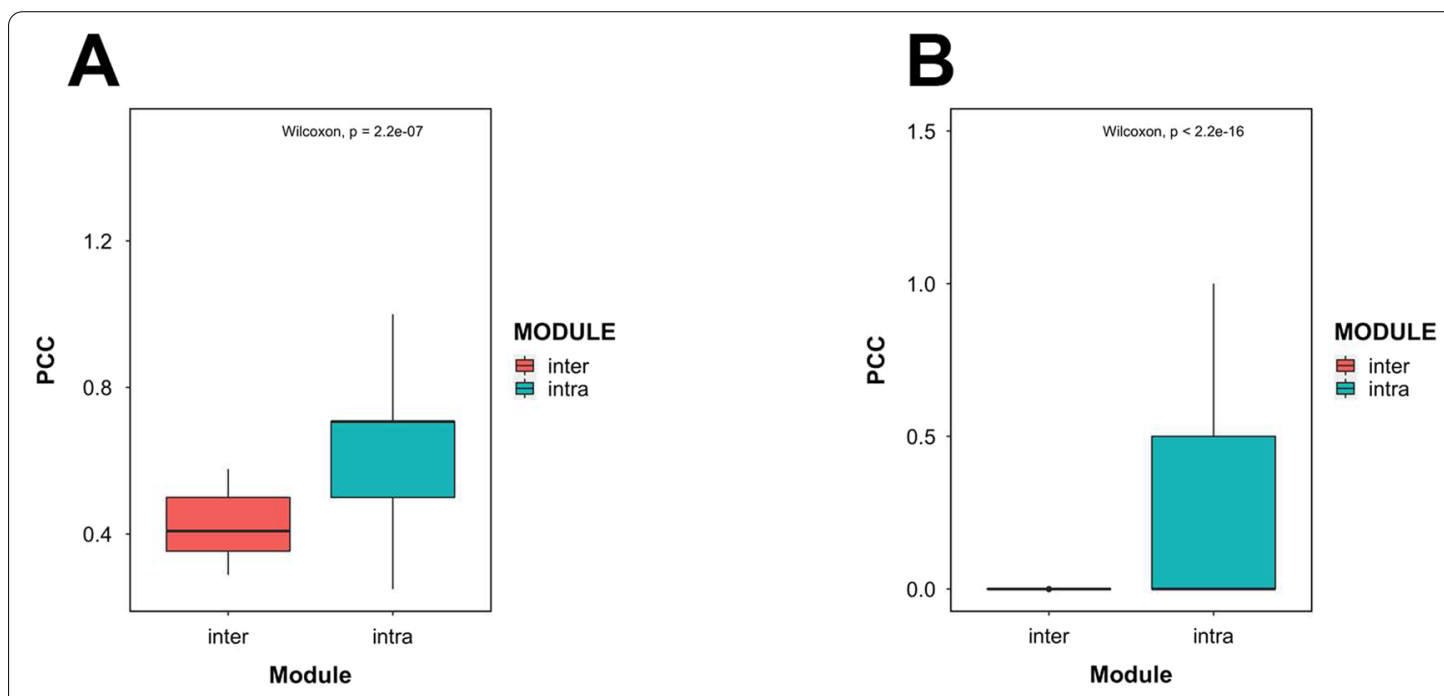

\section{C}

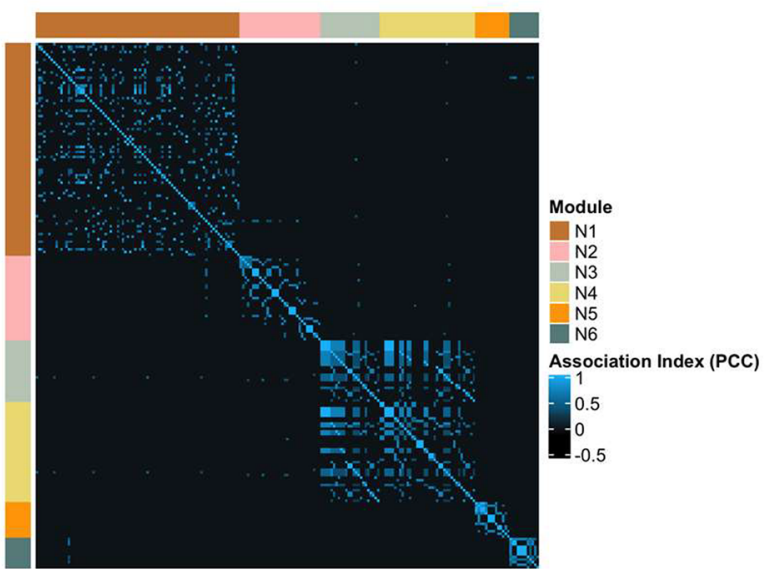

D

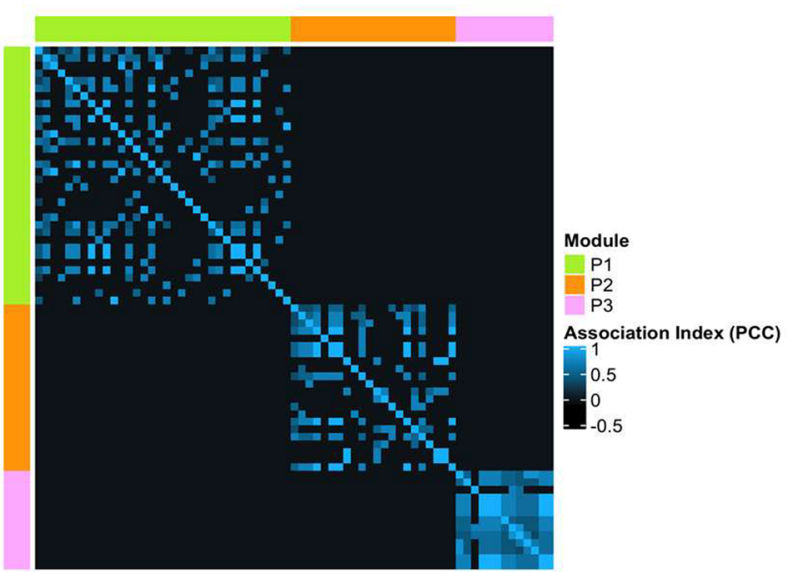

Fig. 5 Comparison of intra-module IncRNAs and inter-module IncRNAs. A, B The PCC between intra-modular IncRNAs and inter-modular IncRNAs were compared in NLAN and PLAN respectively. Wilcoxon test was used to compare two groups. C, D The PCCs of each IncRNA were visualized in heatmaps. The clusters in heatmpas were highly correspond to IncRNA modules identified in the association networks 
Table 1 Survival-related IncRNAs in each prognostic IncRNA module and the overall survival

\begin{tabular}{llll}
\hline ID & Name & Module & Survival \\
\hline ENSG00000258365 & AC073655.2 & N1 & Favourable \\
ENSG00000272402 & AL031775.2 & & Favourable \\
ENSG00000230163 & AL122010.1 & & Favourable \\
ENSG00000267461 & AC079210.1 & & Favourable \\
ENSG00000273226 & AL391834.2 & & Favourable \\
ENSG00000232611 & AL683813.2 & N2 & Favourable \\
ENSG00000268650 & AC005759.1 & & Favourable \\
ENSG00000271828 & AC008937.3 & N4 & Favourable \\
ENSG00000271646 & AC099343.4 & P3 & Favourable \\
\hline
\end{tabular}

lncRNA modules at all time points. The highest AUC of the 4-module lncRNA signature appears on the 336th day $(\mathrm{AUC}=(0.68))$.

\section{The IncRNA modules are related to cancer hallmarks}

Until recently, functions of many lncRNAs in cancer have still not been fully characterized [22]. We used preranked gene set enrichment analysis to deduce the functions of the prognostic lncRNA modules [23]. It was found that Myc targets, E2F targets, and cell cycle were enriched in module N1, N2 and P3. Further, in module $\mathrm{N} 4$, inflammation/immunity-related terms, androgen/ oestrogen response, and KRAS signalling were revealed. Of note, several pathways of other cancer types were enriched in the module P3, implying the involvement of other cancer types as well (Fig. 8). Some of our enrichment results were consistent with the findings validated in other published literatures, where the expression of the lncRNAs were linked to cancer-related processes such as E2F [24], c-Myc [25, 26], androgen receptor [27, 28 ] and oestrogen receptor [29]. All in all, the enrichment analysis revealed cancer hallmarks in which lncRNA modules involve. The enriched terms not only reproduce the characters of lncRNAs found previously, but as well reveal several novel mechanisms in which lncRNA modules may participate.

\section{Discussion}

This study utilized an association network approach to group similar lncRNAs, identify four prognosis-related lncRNA modules and assess the performance of the modular signature as a novel biomarker in LUAD. Biologically, these lncRNA modules involve in various oncogenic mechanisms. Altogether, this study not only revealed the roles of lncRNA modules but also came up with a new module-based biomarker in LAUD.
The genetic regulation of lncRNA is complex. Thus far, we merely made a correlation and association analyses to model the relation between lncRNAs and mRNAs. It may not be proper to infer the causal relationship between lncRNAs and mRNAs from this study. In fact, lncRNA can regulate mRNAs through cis- and trans- regulation, while mRNAs can also regulate the expression of lncRNAs through other mechanisms [30,31]. The direction of regulation between lncRNAs and mRNAs still need further investigation in the future.

Numerous studies also focused on the issue regarding the competing endogenous RNA (ceRNA) networks [3234]. Via constructing a IncRNA-miRNA-mRNA ceRNA network, the molecular mechanisms of certain diseases can be further clarified. A LUAD-specific ceRNA network revealed that seven lncRNAs were associated with the prognosis through interacting with one miRNA (hsa-mir-31) and 16 mRNAs [35]. Zhang et al. identified a LUAD-specific ceRNA network through curating the experiment-supported databases. Based on this ceRNA network, they used random walking and restart algorithm to rank lncRNAs that are associated with LUAD. An in vitro validation was subsequently conducted to prove their prediction that MAPKAPK5-AS1, a novel LUAD-related IncRNA, plays crucial roles in tumour growth [36]. Compared with the construction of ceRNA network in LUAD, our approach mainly used the correlation between lncRNAs and mRNAs to infer the association network of lncRNAs, without considering the roles of miRNAs. However, the advantages of our study include using the real-world data to assess the impact of the lncRNA signature on patients' survival, and evaluating the lncRNA signature as a biomarker in LUAD.

Network biology has become popular in biomedical researches. Analysing networks can model the complex interactions of the biomolecules, providing more accurate predictions [19, 21, 37-41]. Furthermore, it was revealed that networks can delineate dissimilar molecules and gather similar nodes as modules [42, 43]. Therefore, in this study, we conjectured that similar lncRNAs would co-appear as modules and are highly associated. We further tested the biological functions of these generic modules, and discovered module-based biomarkers in LUAD. It is not denying that surveying the biomarkers within the IncRNA association networks may miss the prognosis-related lncRNAs that are not appear in the networks. However, the goal of this study was to construct a LUADspecific lncRNA association network and assess whether these modules play important roles in LUAD, instead of revealing all lncRNAs related to the prognosis in LUAD.

One of the future applications of this study is that the distance of modules in the networks can be quantified by the shortest path length (proximity), which was proved to 

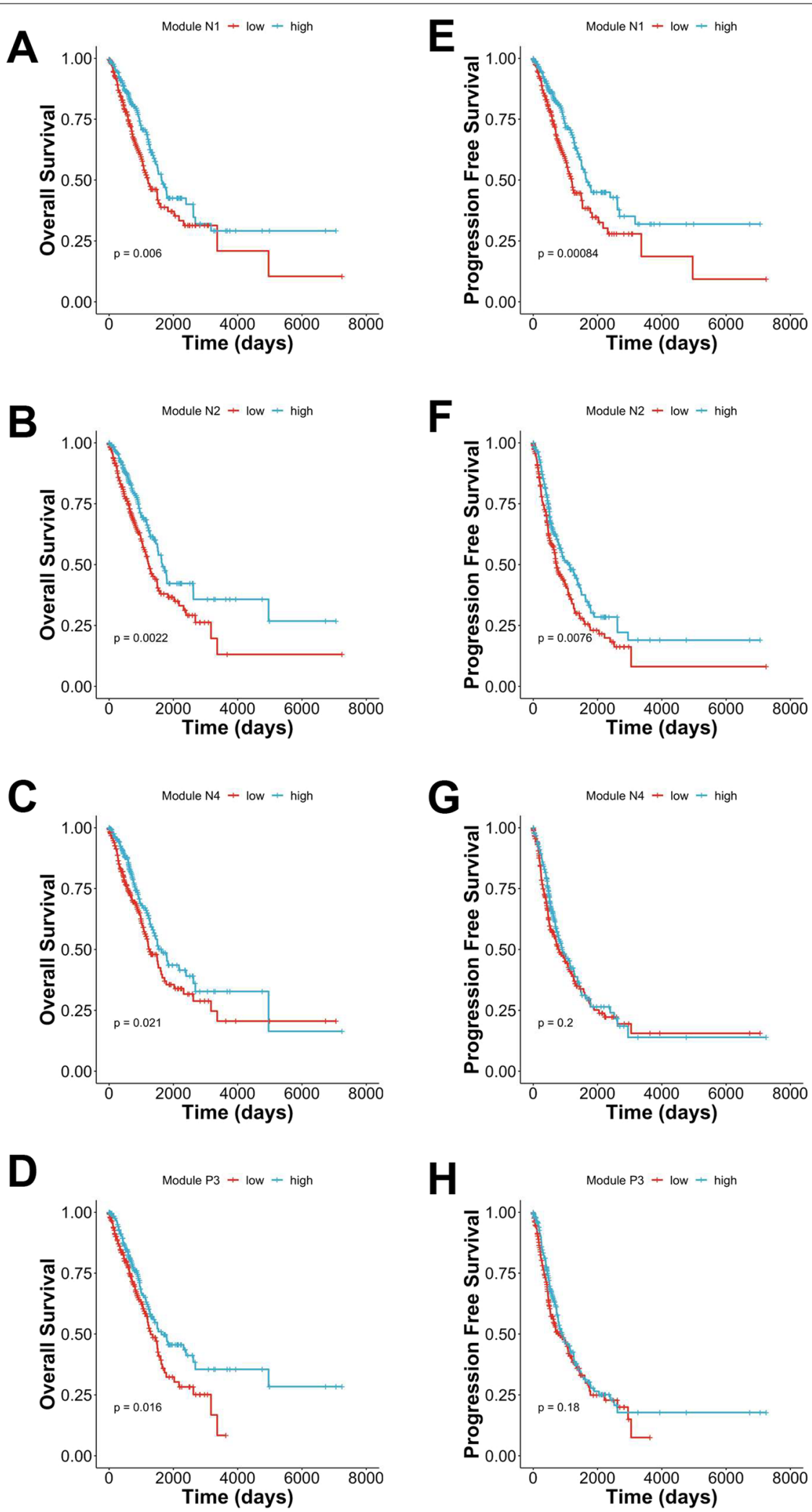

Fig. 6 Survival analysis of prognostic IncRNA modules. The median of modular gene expression was used to assign patients into high or low expression groups. Log-rank test was used to test statistical significance. A-D. Kaplan-Meier curve of the overall survival (OS) in module N1, N2, N4, and P3. E-G. Kaplan-Meier curve of the event-free survival (EFS) in module N1, N2, N4, and P3 
be a successful method to judge the relatedness between clusters of nodes [38]. This fact can be further applied to the cancer drug repurposing. For instance, a recent study found that the proximity between disease-related proteins and drug targets in the protein interactome is an effective measure to predict the response to the drugs [39]. The accuracy of the proximity method even outweigh the traditional expression-based perturbation methods, such as The Library of Integrated Network-Based Cellular Signatures (LINCS) [39]. In reality, according to the latest LUAD guideline [3], targeting lncRNA modules is not feasible. Therefore, the drug repurposing method that interrogating the proximity between IncRNA module-related proteins and drug targets may be one of the solutions.

We referred our findings to The Cancer LncRNome Atlas [44], a database collecting lncRNA alterations across multiple human cancer types (http://fcgportal.org/TCLA/ search.php). It can be seen that the lncRNAs revealed in this study were novel. Copy number variation $(\mathrm{CNV})$ deletion were found in AC073655.2 and AC099343.4, but the alterations in expression level have been unclear. Four lncRNAs (AC008937.3, AL683813.2, AL031775.2, AL122010.1, and AC005759.1) were detectable in LUAD. The other two lncRNAs, AL391834.2, and AC079210.1, were not detectable in LUAD (Additional file 1: Table S2). It is believed that gene expression can be affected by CNV, where decreased gene expression may be attributed to deletions [45]. In our study, we found that decreased expression of AC073655.2 and AC099343.4 are associated with poorer prognosis in LUAD patients. Therefore, it may not be inappropriate to postulate that the underlying mechanisms might be associated with the deletions. However, further investigations will be needed to validate this hypothesis.

\section{Conclusions}

In conclusion, by mining the expression profile from TCGA, we constructed lncRNA-association networks and revealed four crucial LUAD-specific lncRNA modules. These modules involve in several cancer-related biological mechanisms and affect patients' survival. The 4-module lncRNA signature can be used as a prognostic biomarker for LUAD. As far as we know, this is the first study using lncRNA association network to discover promising lncRNA modular biomarkers in LUAD. This method offered a novel insight into the co-regulated lncRNAs in lung cancer and may become a framework for future studies, such as the lncRNA module-based target therapy.

\section{Methods \\ Pre-processing of IncRNA and mRNA expression profiles}

We retrieved the transcriptome data of lung adenocarcinoma (LUAD) from Genomic data commons (GDC) data portal. The lncRNA and mRNA expression profile were collected from the HTSeq-FPKM-UQ workflow of TCGALUAD project $(n=553)$ in February 2018. The pre-processing procedure of the gene expression profile was adjusted from our previous work [21]. In brief, genes with more than $20 \%$ of values to be NAs were removed. Next, the remaining NAs were filled with 0 . We then adjusted the expression of genes with $\log 2$ transformation. To link the Ensembl gene ID with gene type, we used Ensembl 92 (GRCh38. p12) as the reference genome. Finally, The Ensembl gene ID was converted to gene symbol by referring to BioMart database [46]. The pre-processed gene expression file contained 4342 lncRNAs and 16618 mRNAs.

We also used identical pre-processing steps to gather the expression profile of adjacent normal tissue for subsequent analyses. These samples were used as control group and pooled from the adjacent normal tissue in both LUAD and lung squamous cell carcinoma (LUSC) project from TCGA, consisting of 108 samples in total.

\section{Identification of LncRNA-correlated mRNAs}

To model the relation between lncRNAs and mRNAs, we used Spearman's correlation coefficient (SCC) (1) to quantify the correlation of lncRNA and mRNA.

$$
S C C=1-\frac{6 \sum d_{i}^{2}}{n\left(n^{2}-1\right)}
$$

where $d_{i}=$ the difference between the two ranks of each investigated gene, and $\mathrm{n}=$ sample size.

The SCCs were then transformed to mutual rank (MR) index. The MR is the geometric average of the SCC rank [21]. That is, for the gene pair lncRNA $X$ and mRNA $Y$, the mutual rank is defined as follow:

$$
M R_{X Y}=\sqrt{\operatorname{Rank}_{X \rightarrow Y} \times \operatorname{Rank}_{Y \rightarrow X}}
$$

In order to select negatively and positively lncRNAcorrelated mRNAs, we ranked the SCCs in ascending and

(See figure on next page.)

Fig. 7 Evaluation of multi-module IncRNA signature. A A forest plot showing the HR and p-value under multivariable Cox PH model to assess overall survival of LUAD patients with high v.s. low expression of the four IncRNA modules (N1, N2, N4, P3) and the 4-module signature. Age and TNM stage were adjusted. B Kaplan-Meier curve of the OS in the 4-module Signature. Log-rank test was used. C Kaplan-Meier curve of the EFS in the 4-module Signature. Log-rank test was used. D The comparison of AUC of module N1, N2, N4, P3 and the 4-module signature in five different time points 
A Module

N1

N2

N4

P3

4-module Signature

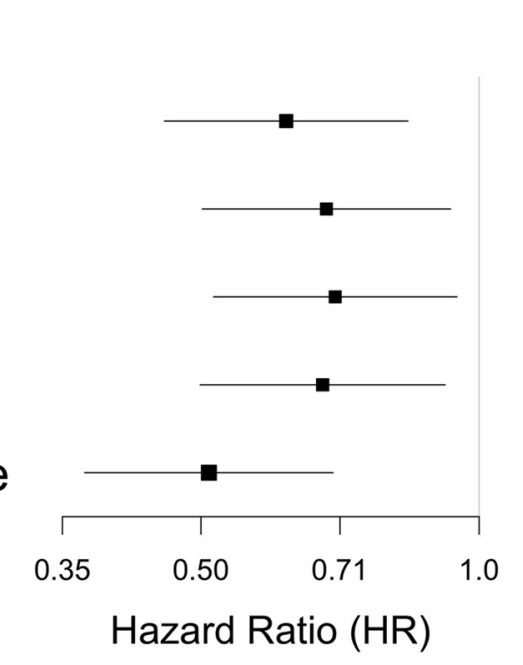

\section{P-value}

0.00189

0.00159

0.0205

0.0123

2.00e-05
B

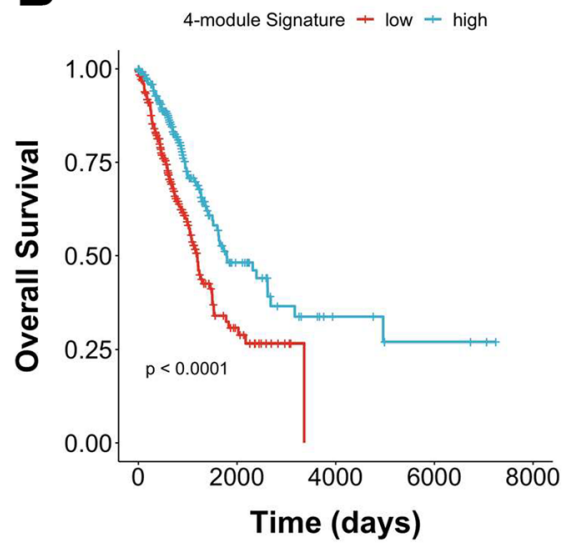

C

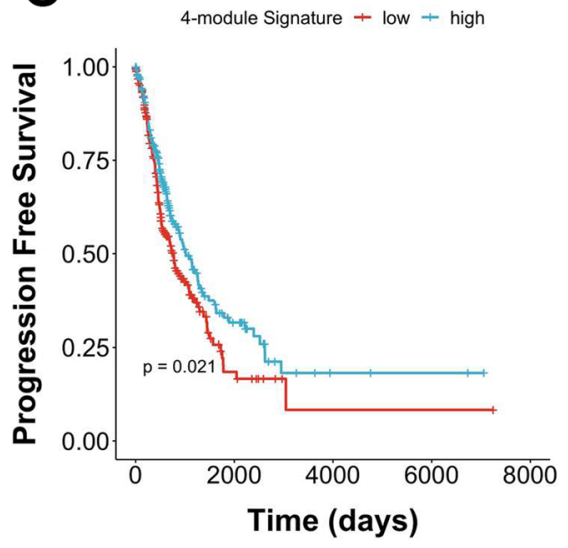

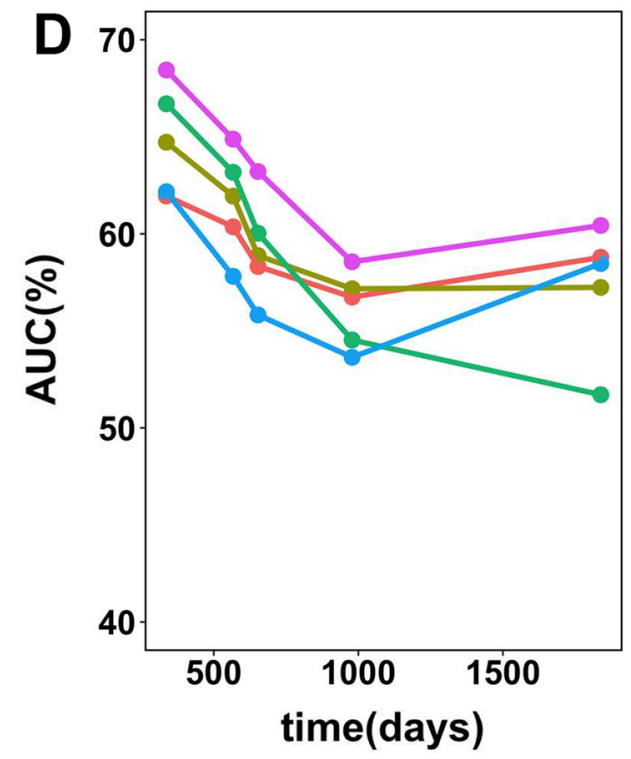

Module

- N1

- N2

- N4

- P3

- 4-module Signature

Fig. 7 (See legend on previous page.) 


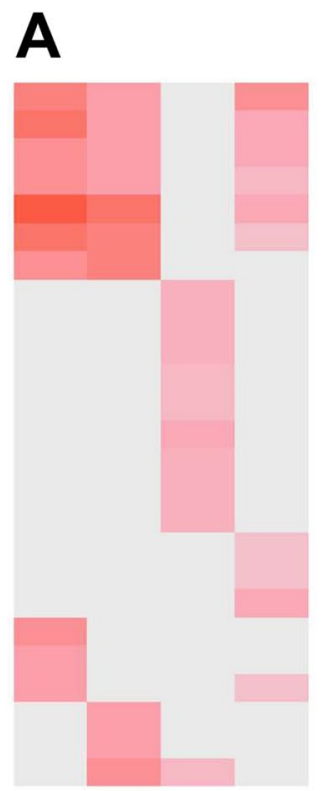

$\Sigma \tilde{z} \frac{\pi}{z}$

B
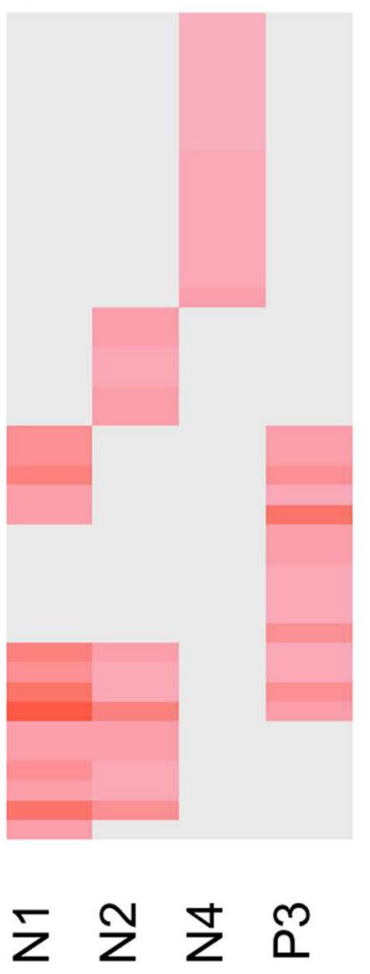

E2F TARGETS

OXIDATIVE PHOSPHORYLATION

G2M_CHECKPOINT

MYC TARGETS V2

MYC ${ }^{-}$TARGETS ${ }^{-} 1$

MTORC1_SIGNALING

EPITHELTAL MESENCHYMAL TRANSITION

COMPLEMENTT

HEDGEHOG_SIGNALING

ESTROGEN RESPONSE EARLY

KRAS SIGNALING UP

INTERFERON ALPHA_RESPONSE

UV RESPONSE DN

INFLAMMATORY RESPONSE

ANDROGEN RESPONSE

TGF BETA SIGNALING

SPERMATOGENESIS

CHOLESTEROL_HOMEOSTASIS

DNA REPAIR

UNFOLDED PROTEIN RESPONSE

PROTEIN SECRETION

GLYCOLYSIS

HYPOXIA

REACTIVE OXYGEN SPECIES PATHWAY

TNFA_SIGNAALING_VTA_NFKB

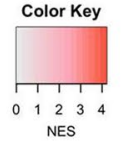

ARRHYTHMOGENIC_RIGHT_VENTRICULAR_CARDIOMYOPATHY_ARVC CELL_ADHESION_MOLECULES_CAMS

FOCĀ_ADHESION

SMALL_CELL_LUNG_CANCER

TOLL LIKE RECEPTOR_SIGNALING_PATHWAY

PANCREATICCEPTOR

CHEMOKINE SIGNALING PATHWAY

HEMATOPOIETIC CELL LINEAGE

O GLYCAN_BIOSYNTTHESIS

PROSTATE CANCER

CYTOKINE CYTOKINE RECEPTOR INTERACTION

ALDOSTERONE REGULATED SODTUM REABSORPTION

PHOSPHATIDYLINOSITOL SIGNALING SYSTEM

COMPLEMENT AND COAGULATION_CASCADES

GALACTOSE METABOLISM

BRDNER

FRUCTOSE AND MANNOSE METABOLISM

GLYCOLYSIS GLUCONEOGENESIS

OXIDATIVE PHOSPHORYLATION

PARKINSONS DISEASE

SPLICEOSOME

ALZHEIMERS DISEASE

DNA REPLICATTION

BASE EXCISION REPAIR

NUCLEOTIDE EXCISIOI

PYRIMIDINE METABOLISM

(1)

HOMOLOGOUS_RECOMBINATION

CELL CYCLE

HUNTINGTONS_DISEASE

PROTEASOME

RIBOSOME

LYSOSOME

PROTEIN EXPORT

PATHOGENIC ESCHERICHIA COLIINFECTION

AMINOACYL TRNA BIOSYNTHESIS

ARGININE AND_PROLINE_METABOLISM

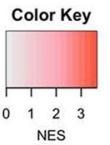

Fig. 8 Comparing functions of prognostic IncRNA modules. A GSEA was used to conduct functional enrichment analysis where top 10 biological processes, based on normalized enrichment score (NES), in each module were shown (FDR q-value $<0.001$ ). B Biological functions regarding KEGG pathways were investigated. Terms with top 15 NES were shown in each module (FDR q-value $<0.001$ ) 
descending order to represent negatively and positively correlated mRNAs respectively. We then defined lncRNAmRNA pairs with top one percent of MR index as highly correlated IncRNA-mRNA pairs.

\section{Construction of IncRNA association networks}

We used the IncRNA mRNA correlation bi-partite networks to deduce the association between lncRNAs. Pearson correlation coefficient (PCC) (3), an association index, was computed to define the association between each lncRNA.

$$
P C C_{X Y}=\frac{|\mathrm{N}(\mathrm{X}) \cap \mathrm{n}(\mathrm{Y})| n_{y}-|\mathrm{N}(\mathrm{X})||\mathrm{N}(\mathrm{Y})|}{\sqrt{|\mathrm{N}(\mathrm{X})||\mathrm{N}(\mathrm{Y})|\left(n_{y}-|\mathrm{N}(\mathrm{X})|\right)\left(n_{y}-|\mathrm{N}(\mathrm{Y})|\right)}}
$$

where $N(X)$ is the number of mRNAs correlated with lncRNA $X, N(Y)$ is the number of genes correlated with lncRNA $\mathrm{Y}, \mathrm{n}_{\mathrm{y}}$ is the number of mRNAs correlated with both lncRNA $X$ and lncRNA Y. We defined two lncRNAs being associated if and only if they shared $\mathrm{PCC}>0.7$. We then collected lncRNA pairs with this criterion into a lncRNA-lncRNA edge list and visualized the lncRNA association network with Cytoscape [47]. The lncRNA association network constructed from negatively correlated mRNAs were coined as negative lncRNA association network (NALN). On the other hand, those considered positively correlated mRNAs were termed positive lncRNA association network (PLAN).

\section{Survival analysis and biomarker assessment}

To select lncRNA modules that determine prognosis of LUAD patients, we designed a three-step selection procedure, implemented with the R package: survival (https:// CRAN.R-project.org/package $=$ survival) and survminer (http://www.sthda.com/english/rpkgs/survminer/). Firstly, the survival time, age at diagnosis, TNM stage, event type (cancer progression or death), and censoring information of TCGA LUAD were collected from clinical data in GDC data portal. We divided patients into two subgroups based on the median of lncRNA expression. The univariate survival analysis was conducted by logrank test. Secondly, to adjust known confounding factors, including patients' age and cancer stage, the genes that passed log-rank test were subsequently examined by multivariable Cox-proportional hazard model. Finally, the remaining lncRNAs without stage-dependency or differential expression were discarded. The stage-dependency analysis was conducted by comparing lncRNA expression across different TNM stage (from stage I to stage IV). Kruskal-Wallis test was used for hypotheses testing.
For the differential expression analysis, we compared the gene expression between cancer and adjacent normal tissue with Wilcoxon test.

For the biomarker assessment, time-dependent area under the receiver operating characteristic (time-AUC) was calculated by using the R package 'time ROC' [48].

\section{Functional gene set enrichment analysis}

To investigate functions of prognostic lncRNA modules, we interrogated IncRNA-correlated mRNAs to conduct pre-ranked GSEA [23]. The mRNAs were ranked based on the correlation (MR index) with the lncRNAs within each module. In PLAN, since the positively correlated mRNAs were more important, the SCCs were arranged in the ascending order, from small to large, which led to higher MR index in the positively correlated mRNAs. By contrast, SCCs were arranged in descending order, which resulted in higher MR index in negatively correlated mRNAs. Gene sets database, Hallmark and KEGG pathway, from MSigDB v7.1 [49, 50] were selected. The chip platform was from Human ENSEMBL_Gene_MSigDB.v7.1.chip. The top 10 and 15 were selected from Hallmark and KEGG pathway respectively. All selected terms were significant, with FDR-q value $<0.001$.

\section{Abbreviations \\ LncRNA: Long non-coding RNA; LUAD: Lung adenocarcinoma; TCGA: The Can- cer Genome Atlas; HR: Hazard ratio; Cl: Confidence interval; AUC: Area under the receiver operating characteristic curve; EMT: Epithelial-mesenchymal transition; SCC: Spearman's correlation coefficient; MR: Mutual rank; PCC: Pear- son correlation coefficient; LAN: LncRNA association network; PLAN: Positive IncRNA association network; NALN: Negative IncRNA association network; OS: Overall survival; PFS: Progression free survival; ceRNA: Competing endogenous RNA; LINCS: The Library of Integrated Network-Based Cellular Signatures; CNV: Copy number variation.}

\section{Supplementary Information}

The online version contains supplementary material available at https://doi. org/10.1186/s12920-021-01137-0.

Additional file 1. Detailed components in each IncRNA module.

\section{Acknowledgements}

We appreciate Hsuan-Ting Huang from Taipei Municipal Jianguo High School for data verification.

\section{About this supplement}

This article has been published as part of BMC Medical Genomics Volume 14 Supplement 3 2021: Selected articles from the 19th Asia Pacific Bioinformatics Conference (APBC 2021): medical genomics The full contents of the supplement are available at https://bmcmedgenomics.biomedcentral.com/articles/ supplements/volume-14-supplement-3.

\section{Authors' contributions}

$\mathrm{HFJ}$ and $\mathrm{HCH}$ conceived, designed, and supervised the study. AL, WHY and $\mathrm{CLH}$ developed the method. AL performed the analyses. AL, HFJ and $\mathrm{HCH}$ wrote the manuscript. All authors read and approved the final manuscript. 


\section{Funding}

This work was supported by the Ministry of Science and Technology, Taiwan (MOST 109-2221-E-002-161-MY3 and MOST 109-2221-E-010-011-MY3) and the Higher Education Sprout Project (NTU-109L8837A). Publication costs are funded by MOST 109-2221-E-002-161-MY3. The funding agencies had no role in the design, analysis, and interpretation of the data or writing of the manuscript.

\section{Availability of data and materials}

The RNA-seq transcriptome profiling of lung adenocarcinoma from TCGA is freely available via GDC data portal (https://portal.gdc.cancer.gov/).

\section{Declarations}

\section{Ethics approval and consent to participate}

Not applicable.

\section{Consent for publication}

Not applicable.

\section{Competing interests}

The authors declare that they have no competing interests.

\section{Author details}

${ }^{1}$ Graduate Institute of Biomedical Electronics and Bioinformatics, National Taiwan University, Taipei 10617, Taiwan. ${ }^{2}$ Department of Medical Research, National Taiwan University Hospital, Taipei 10002, Taiwan. ${ }^{3}$ Institute of Biomedical Informatics, National Yang Ming Chiao Tung University, Taipei 11221, Taiwan. ${ }^{4}$ Department of Life Science, National Taiwan University, Taipei 10617, Taiwan. ${ }^{5}$ Center for Computational and Systems Biology, National Taiwan University, Taipei 10617, Taiwan.

Received: 24 November 2021 Accepted: 30 November 2021 Published online: 06 December 2021

\section{References}

1. Fitzmaurice C, Abate D, Abbasi N, Abbastabar H, Abd-Allah F, AbdelRahman O, Abdelalim A, Abdoli A, Abdollahpour I, Abdulle ASM, et al. Global, regional, and national cancer incidence, mortality, years of life lost, years lived with disability, and disability-adjusted life-years for 29 cancer groups, 1990 to 2017: a systematic analysis for the global burden of disease study. JAMA Oncol. 2019:5(12):1749-68.

2. Reck M, Rabe KF. Precision diagnosis and treatment for advanced nonsmall-cell lung cancer. N Engl J Med. 2017:377(9):849-61.

3. Non-small Cell Lung Cancer (Version 6, 2020) https://www.nccn.org/ professionals/physician_gls/pdf/nscl.pdf.

4. Ma L, Bajic VB, Zhang Z. On the classification of long non-coding RNAs. RNA Biol. 2013;10(6):925-33.

5. Martens JA, Laprade L, Winston F. Intergenic transcription is required to repress the Saccharomyces cerevisiae SER3 gene. Nature. 2004:429(6991):571-4.

6. Martianov I, Ramadass A, Serra Barros A, Chow N, Akoulitchev A. Repression of the human dihydrofolate reductase gene by a non-coding interfering transcript. Nature. 2007;445(7128):666-70.

7. Hirota K, Miyoshi T, Kugou K, Hoffman CS, Shibata T, Ohta K. Stepwise chromatin remodelling by a cascade of transcription initiation of noncoding RNAs. Nature. 2008;456(7218):130-4.

8. Pandey GK, Mitra S, Subhash S, Hertwig F, Kanduri M, Mishra K, Fransson S, Ganeshram A, Mondal T, Bandaru S, et al. The risk-associated long noncoding RNA NBAT-1 controls neuroblastoma progression by regulating cell proliferation and neuronal differentiation. Cancer Cell. 2014;26(5):722-37.

9. Marín-Béjar O, Marchese FP, Athie A, Sánchez Y, González J, Segura V, Huang L, Moreno I, Navarro A, Monzó M, et al. Pint lincRNA connects the $\mathrm{p} 53$ pathway with epigenetic silencing by the Polycomb repressive complex 2. Genome Biol. 2013;14(9):R104.
10. Yoon JH, Abdelmohsen K, Srikantan S, Yang X, Martindale JL, De S, Huarte M, Zhan M, Becker KG, Gorospe M. LincRNA-p21 suppresses target mRNA translation. Mol Cell. 2012:47(4):648-55.

11. Alaei S, Sadeghi B, Najafi A, Masoudi-Nejad A. LncRNA and mRNA integration network reconstruction reveals novel key regulators in esophageal squamous-cell carcinoma. Genomics. 2019;111(1):76-89.

12. Jiang $L$, Hong L, Yang W, Zhao Y, Tan A, Li Y. Co-expression network analysis of the IncRNAs and mRNAs associated with cervical cancer progression. Arch Med Sci. 2019;15(3):754-64.

13. Zhang H, Zhu M, Du Y, Zhang H, Zhang Q, Liu Q, Huang Z, Zhang L, Li H, $\mathrm{Xu} L$, et al. A panel of 12-IncRNA signature predicts survival of pancreatic adenocarcinoma. J Cancer. 2019;10(6):1550-9.

14. Li J, Chen Z, Tian L, Zhou C, He MY, Gao Y, Wang S, Zhou F, Shi S, Feng X, et al. LncRNA profile study reveals a three-IncRNA signature associated with the survival of patients with oesophageal squamous cell carcinoma. Gut. 2014;63(11):1700-10

15. Zhou M, Zhang Z, Bao S, Hou P, Yan C, Su J, Sun J. Computational recognition of IncRNA signature of tumor-infiltrating B lymphocytes with potential implications in prognosis and immunotherapy of bladder cancer. Brief Bioinform. 2020. https://doi.org/10.1093/bib/bbaa047.

16. Ma B, Li Y, Ren Y. Identification of a 6-IncRNA prognostic signature based on microarray re-annotation in gastric cancer. Cancer Med. 2020;9(1):335-49.

17. Tang J, Ren J, Cui Q, Zhang D, Kong D, Liao X, Lu M, Gong Y, Wu G. A prognostic 10-IncRNA expression signature for predicting the risk of tumour recurrence in breast cancer patients. J Cell Mol Med. 2019;23(10):6775-84.

18. Li J, Wang W, Xia P, Wan L, Zhang L, Yu L, Wang L, Chen X, Xiao Y, Xu C. Identification of a five-IncRNA signature for predicting the risk of tumor recurrence in patients with breast cancer. Int J Cancer. 2018;143(9):2150-60

19. Fuxman Bass Jl, Diallo A, Nelson J, Soto JM, Myers CL, Walhout AJM. Using networks to measure similarity between genes: association index selection. Nat Methods. 2013;10(12):1169-76.

20. Oliver S. Guilt-by-association goes global. Nature. 2000;403(6770):601-3.

21. Wu CH, Hsu CL, Lu PC, Lin WC, Juan HF, Huang HC. Identification of IncRNA functions in lung cancer based on associated protein-protein interaction modules. Sci Rep. 2016;6:35939.

22. Carlevaro-Fita J, Lanzós A, Feuerbach L, Hong C, Mas-Ponte D, Pedersen JS, Abascal F, Amin SB, Bader GD, Barenboim J, et al. Cancer LncRNA Census reveals evidence for deep functional conservation of long noncoding RNAs in tumorigenesis. Commun Biol. 2020;3(1):56.

23. Subramanian A, Tamayo P, Mootha VK, Mukherjee S, Ebert BL, Gillette MA Paulovich A, Pomeroy SL, Golub TR, Lander ES, et al. Gene set enrichment analysis: a knowledge-based approach for interpreting genome-wide expression profiles. Proc Natl Acad Sci U S A. 2005;102(43):15545-50.

24. Feldstein O, Nizri T, Doniger T, Jacob J, Rechavi G, Ginsberg D. The long non-coding RNA ERIC is regulated by E2F and modulates the cellular response to DNA damage. Mol Cancer. 2013;12(1):131.

25. Hart JR, Roberts TC, Weinberg MS, Morris KV, Vogt PK. MYC regulates the non-coding transcriptome. Oncotarget. 2014;5(24):12543-54.

26. Kim T, Jeon YJ, Cui R, Lee JH, Peng Y, Kim SH, Tili E, Alder H, Croce CM. Role of MYC-regulated long noncoding RNAs in cell cycle regulation and tumorigenesis. J Natl Cancer Inst. 2015. https://doi.org/10.1093/jnci/ dju505

27. Wang LG, Johnson EM, Kinoshita Y, Babb JS, Buckley MT, Liebes LF, Melamed J, Liu XM, Kurek R, Ossowski L, et al. Androgen receptor overexpression in prostate cancer linked to Pur alpha loss from a novel repressor complex. Cancer Res. 2008;68(8):2678-88.

28. Takayama K, Horie-Inoue K, Katayama S, Suzuki T, Tsutsumi S, Ikeda K, Urano T, Fujimura T, Takagi K, Takahashi S, et al. Androgen-responsive long noncoding RNA CTBP1-AS promotes prostate cancer. EMBO J. 2013;32(12):1665-80.

29. Chakravarty D, Sboner A, Nair SS, Giannopoulou E, Li R, Hennig S, Mosquera JM, Pauwels J, Park K, Kossai M, et al. The oestrogen receptor alpha-regulated IncRNA NEAT1 is a critical modulator of prostate cancer. Nat Commun. 2014:5:5383.

30. Sánchez Y, Segura V, Marín-Béjar O, Athie A, Marchese FP, González J, Bujanda L, Guo S, Matheu A, Huarte M. Genome-wide analysis of the human p53 transcriptional network unveils a IncRNA tumour suppressor signature. Nat Commun. 2014;5:5812. 
31. Huarte M. The emerging role of IncRNAs in cancer. Nat Med. 2015;21(11):1253-61.

32. Cong Z, Diao Y, Xu Y, Li X, Jiang Z, Shao C, Ji S, Shen Y, De W, Qiang Y. Long non-coding RNA linc00665 promotes lung adenocarcinoma progression and functions as ceRNA to regulate AKR1B10-ERK signaling by sponging miR-98. Cell Death Dis. 2019;10(2):84.

33. Salmena L, Poliseno L, Tay Y, Kats L, Pandolfi Pier P. A ceRNA hypothesis: the Rosetta stone of a hidden RNA language? Cell. 2011;146(3):353-8.

34. Tay Y, Rinn J, Pandolfi PP. The multilayered complexity of ceRNA crosstalk and competition. Nature. 2014;505(7483):344-52.

35. Wu X, Sui Z, Zhang H, Wang Y, Yu Z. Integrated Analysis of IncRNAMediated ceRNA Network in Lung Adenocarcinoma. Front Oncol. 2020;10:554759

36. Zhang $\mathrm{H}$, Wang $\mathrm{Y}$, Lu J. Identification of lung-adenocarcinoma-related long non-coding RNAs by random walking on a competing endogenous RNA network. Ann Transl Med. 2019;7(14):339.

37. Barabási AL, Oltvai ZN. Network biology: understanding the cell's functional organization. Nat Rev Genet. 2004;5(2):101-13.

38. Menche J, Sharma A, Kitsak M, Ghiassian SD, Vidal M, Loscalzo J, Barabási A-L. Disease networks. Uncovering disease-disease relationships through the incomplete interactome. Science (New York, NY). 2015;347(6224):1257601.

39. Guney E, Menche J, Vidal M, Barábasi AL. Network-based in silico drug efficacy screening. Nat Commun. 2016;7:10331.

40. Cheng F, Kovács IA, Barabási AL. Network-based prediction of drug combinations. Nat Commun. 2019;10(1):1197.

41. Barabási AL, Gulbahce N, Loscalzo J. Network medicine: a network-based approach to human disease. Nat Rev Genet. 2011;12(1):56-68.

42. van Dam S, Võsa U, van der Graaf A, Franke L, de Magalhães JP. Gene co-expression analysis for functional classification and gene-disease predictions. Brief Bioinform. 2018;19(4):575-92.

43. Langfelder P, Horvath S. WGCNA: an R package for weighted correlation network analysis. BMC Bioinform. 2008;9:559.

44. Yan X, Hu Z, Feng Y, Hu X, Yuan J, Zhao SD, Zhang Y, Yang L, Shan W, He Q, et al. Comprehensive genomic characterization of long non-coding RNAs across human cancers. Cancer Cell. 2015;28(4):529-40,

45. Zhou C, Zhang W, Chen W, Yin Y, Atyah M, Liu S, Guo L, Shi Y, Ye Q, Dong $\mathrm{Q}$, et al. Integrated analysis of copy number variations and gene expression profiling in hepatocellular carcinoma. Sci Rep. 2017;7(1):10570.

46. Smedley D, Haider S, Ballester B, Holland R, London D, Thorisson G, Kasprzyk A. BioMart-biological queries made easy. BMC Genom. 2009;10:22-22

47. Shannon P, Markiel A, Ozier O, Baliga NS, Wang JT, Ramage D, Amin $\mathrm{N}$, Schwikowski B, Ideker T. Cytoscape: a software environment for integrated models of biomolecular interaction networks. Genome Res. 2003;13(11):2498-504.

48. Blanche P, Dartigues JF, Jacqmin-Gadda H. Estimating and comparing time-dependent areas under receiver operating characteristic curves for censored event times with competing risks. Stat Med. 2013;32(30):5381-97.

49. Liberzon A, Subramanian A, Pinchback R, Thorvaldsdóttir H, Tamayo P, Mesirov JP. Molecular signatures database (MSigDB) 3.0. Bioinformatics. 2011;27(12):1739-40.

50. Liberzon A, Birger C, Thorvalds dóttir H, Ghandi M, Mesirov JP, Tamayo P. The molecular signatures database (MSigDB) hallmark gene set collection. Cell Syst. 2015;1(6):417-25.

\section{Publisher's Note}

Springer Nature remains neutral with regard to jurisdictional claims in published maps and institutional affiliations.

Ready to submit your research? Choose BMC and benefit from:

- fast, convenient online submission

- thorough peer review by experienced researchers in your field

- rapid publication on acceptance

- support for research data, including large and complex data types

- gold Open Access which fosters wider collaboration and increased citations

- maximum visibility for your research: over $100 \mathrm{M}$ website views per year

At BMC, research is always in progress.

Learn more biomedcentral.com/submissions 